\title{
Erratum to: Hydrochemistry and evaluation of groundwater suitability for irrigation and drinking purposes in the Markandeya River basin, Belgaum District, Karnataka State, India
}

\section{P. Ravikumar • R. K. Somashekar • Mhasizonuo Angami}

Published online: 28 April 2010

(C) Springer Science+Business Media B.V. 2010

\section{Erratum to: Environ Monit Assess}

\section{DOI 10.1007/s10661-010-1399-2}

The original version of this article unfortunately contained mistake in Table 1 and Eqs. 1 and 5.

The corrected Table 1 and equations are given below:

$$
\begin{gathered}
E=\frac{\sum \text { cations }-\sum \text { anions }}{\sum \text { cations }+\sum \text { anions }} \times 100 \\
\mathrm{SSP}=\frac{\mathrm{Na}^{+}}{\left(\mathrm{Na}^{+}+\mathrm{Ca}^{2+}+\mathrm{Mg}^{2+}\right)} \times 100
\end{gathered}
$$


Table 1 Physico-chemical and irrigation quality parameters with BIS standards

\begin{tabular}{|c|c|c|c|c|c|}
\hline Sl. no & $\begin{array}{l}\text { Category of } \\
\text { parameters }\end{array}$ & Characteristics & Analytical method & Unit & $\begin{array}{l}\text { BIS Max. } \\
\text { permissible } \\
\text { limit (1998) }\end{array}$ \\
\hline 1 & General & $\mathrm{pH}$ & Electrode & - & $6.5-8.5$ \\
\hline 2 & & Redox potential (Eh) & Electrode & $\mathrm{mV}$ & NA \\
\hline 3 & & EC & Conductivity-TDS meter & $\mu \mathrm{S} / \mathrm{cm}$ & 3,000 \\
\hline 4 & & TDS & Conductivity-TDS meter & $\mathrm{mg} / \mathrm{L}$ & 2,000 \\
\hline 5 & & Total alkalinity (as $\left.\mathrm{CaCO}_{3}\right)$ & Titrimetric & $\mathrm{mg} / \mathrm{L}$ & 600 \\
\hline 6 & & Temperature & Electrode & ${ }^{\circ} \mathrm{C}$ & NA \\
\hline 7 & & Total hardness (as $\mathrm{CaCO}_{3}$ ) & EDTA titrimetric & $\mathrm{mg} / \mathrm{L}$ & 600 \\
\hline 8 & & Calcium hardness $\left(\right.$ as $\left.\mathrm{CaCO}_{3}\right)$ & EDTA titrimetric & $\mathrm{mg} / \mathrm{L}$ & 200 \\
\hline 9 & & Color & Colorimetric & Hazens & 25 \\
\hline 10 & & Turbidity & Colorimetric & NTU & 10 \\
\hline 11 & Major cations & Calcium $\left(\right.$ as $\left.\mathrm{Ca}^{2+}\right)$ & EDTA titrimetric & $\mathrm{mg} / \mathrm{L}$ & 200 \\
\hline 12 & & 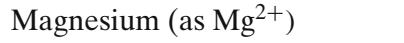 & EDTA titrimetric & $\mathrm{mg} / \mathrm{L}$ & 100 \\
\hline 13 & & Sodium $\left(\right.$ as $\left.\mathrm{Na}^{+}\right)$ & Flame photometric & $\mathrm{mg} / \mathrm{L}$ & 200 \\
\hline 14 & & Potassium (as $\left.\mathrm{K}^{2+}\right)$ & & $\mathrm{mg} / \mathrm{L}$ & 10 \\
\hline 15 & Major anions & Bicarbonates (as $\mathrm{HCO}_{3}^{-}$) & Titrimetric & $\mathrm{mg} / \mathrm{L}$ & NA \\
\hline 16 & & Carbonates (as $\mathrm{CO}_{3}^{2-}$ ) & Titrimetric & $\mathrm{mg} / \mathrm{L}$ & NA \\
\hline 17 & & Chlorides & Argentometric & $\mathrm{mg} / \mathrm{L}$ & 1,000 \\
\hline 18 & & Nitrates $\left(\right.$ as $\mathrm{NO}_{3}^{-}$) & ISE (ion selective electrode) & $\mathrm{mg} / \mathrm{L}$ & 45 \\
\hline 19 & & Fluoride $\left(\right.$ as $\left.\mathrm{F}^{-}\right)$ & & $\mathrm{mg} / \mathrm{L}$ & 1.5 \\
\hline 20 & & Phosphates (as $\mathrm{PO}_{4}^{3-}$ ) & Stannous chloride & $\mathrm{mg} / \mathrm{L}$ & 0.3 \\
\hline 21 & & Sulfates $\left(\right.$ as $\left.\mathrm{SO}_{4}^{2-}\right)$ & Barium chloride & $\mathrm{mg} / \mathrm{L}$ & 400 \\
\hline 23 & $\begin{array}{l}\text { Irrigation water } \\
\text { quality }\end{array}$ & Boron (B) & Curcumin method & $\begin{array}{c}\mathrm{mg} / \mathrm{L} \text { or } \\
\mu \mathrm{g} / \mathrm{L}\end{array}$ & - \\
\hline 24 & & Hardness (as $\mathrm{CaCO}_{3}$ ) & By calculation using equations & $\mathrm{mg} / \mathrm{L}$ & $<75$ \\
\hline 25 & & Salinity & & $\%$ & NA \\
\hline 26 & & SAR & & - & $<10$ or $10-18$ \\
\hline 27 & & RSC & & $\mathrm{meq} / \mathrm{L}$ & $<1.25$ \\
\hline 28 & & RSBC & & meq/L & $<5 \mathrm{mg} / \mathrm{L}$ \\
\hline 29 & & $\% \mathrm{Na}$ & & $\%$ & $<20$ or $20-40$ \\
\hline 30 & & PI & & $\%$ & Class I or II \\
\hline 31 & & $\mathrm{KI}$ & & - & $<1.0$ \\
\hline 32 & & PS & & $\mathrm{meg} / \mathrm{L}$ & NA \\
\hline 33 & & $\mathrm{MH}$ & & $\%$ & below $50 \%$ \\
\hline 34 & & $\mathrm{MR}(\mathrm{Mg} / \mathrm{Ca})$ & & - & $<1.5$ \\
\hline 35 & & CAI- 1 and CAI-2 & & - & $\begin{array}{l}\text { +ve or - ve } \\
\text { values }\end{array}$ \\
\hline 36 & & SSP & & $\%$ & NA \\
\hline 37 & & ESR & & - & NA \\
\hline
\end{tabular}

$N A$ not available 